\title{
海水处理下菊芋幼苗生理生化特性及磷效应的研究
}

\author{
隆小华 刘兆普* 徐文君 \\ （南京农业大学资源与环境学院, 南京 210095)
}

摘 要 种植抗盐耐海水植物是合理利用和开发海涂资源的有效措施之一。该试验研究了海水处理下菊芋 ( Helianthus tuberosus) 幼苗生长发育、渗透物质积累、保护性酶活性、膜透性和离子吸收分布情况及磷素对其影响。结果 表明: 1) $10 \%$ 海水对菊芋幼苗生长发育没有抑制作用, 甚至有一定的促进作用, $25 \%$ 海水胁迫对菊芋幼苗形态发育 上具有一定抑制作用, 增加磷素浓度后, 能显著缓解其抑制作用;2) $10 \%$ 和 $25 \%$ 海水处理下, 脯氨酸和可溶性糖含 量较对照显著增加, 随着时间的延长, 先增加后降低, 增加磷素浓度后, 能显著增加菊芋幼苗叶片脯氨酸和可溶性 糖含量; 3)海水处理下菊芋幼苗叶片 SOD、POD 和 CAT 活性都显著增加, 增加磷素浓度后, 能显著增强菊芋幼苗叶 片 SOD、POD 和 CAT 活性; 4) $10 \%$ 海水处理菊芋幼苗叶片 MDA 含量与对照差异不大, 甚至小于对照, $25 \%$ 海水处理 能显著增加 MDA 含量及膜透性, 增加磷素浓度后, 均降低了 MDA 含量和膜透性; 5) 随着海水浓度增加和时间延长, 菊芋幼苗地上部和根部 $\mathrm{Na}^{+}$和 $\mathrm{Cl}^{-}$含量显著增加, 增加磷素浓度后, 均能降低地上部和根部 $\mathrm{Na}^{+}$和 $\mathrm{Cl}^{-}$含量, 而地 上部和根部 $\mathrm{K}^{+} 、 \mathrm{Ca}^{2+}$ 和 $\mathrm{Mg}^{2+}$ 含量较对照增加, 增加磷素浓度后, 均能增加 $\mathrm{K}^{+} 、 \mathrm{Ca}^{2+}$ 和 $\mathrm{Mg}^{2+}$ 含量。由此可见, 磷素 能够改善菊芋幼苗的营养状况, 同时能够增强其抗盐性。

关键词 菊芋 磷效应 海水处理 渗透物质 保护酶 离子

\section{EFFECTS OF SEAWATER TREATMENTS ON THE PHYSIOLOGICAL AND BIOCHEMICAL CHARACTERISTICS OF HELIANTHUS TUBEROSUS SEEDLINGS AND RESPONSE TO PHOSPHORUS SUPPLY}

\author{
LONG Xiao-Hua LIU Zhao-Pu and XU Wen-Jun \\ ( College of Resources and Environmental Sciences of NAU, Nanjing 210095; China)
}

\begin{abstract}
Growing plants that are tolerant to salt-water is one option for exploiting mudflats along the coast. Pot experiments were carried out to study the effects of seawater on growth, inorganic and organic osmotic, antioxidant enzyme, membrane permeability in Helianthus tuberosus seedlings and response to phosphorus supply. The results showed that 1) compared with the control, the fresh weights of roots and aerial parts of Helianthus tuberosus seedlings treated with $10 \%$ seawater changed slightly, even were higher than the control, whereas decreased with treatment of $25 \%$ seawaters. The fresh weights of roots and aerial parts increased with the concentration of phosphorus augment. The trends of dry weights of roots and aerial parts resembled the trends of fresh weights with the same treatments. 2) The contents of proline and soluble-sugar increased obviously with increasing seawater concentrations. The contents of proline and soluble-sugar increased on 8 days and decreased on 12 days after the treatments of $10 \%$ and $25 \%$ seawater. They both increased with phosphorus augment. 3) The activities of SOD, POD and CAT in leaves of seawater-stressed plants were significantly stimulated compared to control plants, and the trends toned up with increasing seawater concentrations. The activities of SOD, POD and CAT all significantly stimulated with phosphorus augment. 4) Compared with the control, there was slight change of MDA content in leaves of Helianthus tuberosus seedlings treated with $10 \%$ seawater. The MDA content and ELP significantly increased with the treatment of $25 \%$ seawater. They both decreased with phosphorus augment. 5) The $\mathrm{Na}^{+}$and $\mathrm{Cl}^{-}$contents of the aerial parts and roots significantly increased with increasing seawater concentrations and time lasting. And the contents of $\mathrm{Na}^{+}$and $\mathrm{Cl}^{-}$decreased with phosphorus augment. Compared with the control, the contents of $\mathrm{K}^{+}, \mathrm{Ca}^{2+}$ and $\mathrm{Mg}^{2+}$ of the aerial parts and roots increased, and they all increased with phosphorus augment. Above all, phosphorus can improve nutrition and increase resisting salinity for Helianthus tuberosus seedlings.
\end{abstract}

Key words Helianthus tuberosus, Effect of phosphorus, Treatment of seawater, Membrane permeability, An- 
tioxidant enzyme, Ions

海水灌溉农业是以海水资源、沿海滩涂资源和 耐盐植物为劳动对象的特殊农业, 它是大农业的一 个新分支。在半干旱或半湿润易旱滨海地区, 用海 水直接灌溉耐盐经济植物, 控制一定的灌溉定额, 采 取有效的调控措施, 土壤盐分与水分会在一个理想 的水平达到平衡, 不会造成土壤严重盐渍化, 而用适 当比例的海水直接灌溉可以大大缓解干旱地区土壤 水分蒸发, 提高土壤保水能力, 这些水分对一些耐盐 植物仍有效, 同时, 海水资源直接用于农业灌溉可以 为海水养殖废水的资源化利用、缓解对海洋的环境 压力, 开辟了一条新途径 (刘兆普等, 2003), 可见发 展海水灌溉农业对经济、社会和生态环境具有重要 作用, 同时对于加快我国农业经济可持续发展, 拓展 农业发展空间具有重要的战略意义( 徐质斌, 2000; 刘兆普等, 2003)。在海水灌溉农业生产实践中, 不 同浓度海水及养分是影响农作物生长发育的主要因 素, 施 P 肥能达到“以磷调盐”、“以磷调水”的效果 (隆小华等, 2005)。但是国内外有关磷对植物耐盐 性的化学调节的报告甚少, 有资料表明, 磷能增强原 生质抵抗脱水的能力, 从而提高一些作物抗旱耐盐 能力 (陆景陵, 1994), 海水胁迫下增磷显著降低芦荟 (Aloe vera) 根系对 $\mathrm{Na}^{+} 、 \mathrm{Cl}^{-}$的吸收、增强幼苗对 $\mathrm{K}^{+} 、 \mathrm{Ca}^{2+}$ 的向上运输, 维持地上部离子平衡, 从而增 强芦荟的耐盐性(邵晶等, 2005)。

菊芋 (Helianthus tuberosus) 为耐寒、耐旱、耐贫 㾑、耐盐碱植物, 菊芋的地上茎叶和地下块茎都是优 良的饲料, 地下块茎含丰富菊糖, 可作蔬菜, 也是重 要能源作物之一(张邦定, 1997; 隆小华等, 2004), 宜 在我国沿海地区海涂大面积种植, 本试验通过对菊
芋幼苗海水处理下磷效应的研究以期为研究海水灌 溉农业及合理施用磷肥提供理论依据。

\section{1 材料和方法}

\section{1 材料的培养和处理}

供试菊芋采于南京农业大学山东莱州“ 863 ”中 试基地。菊芋块茎用自来水及蒸馏水冲洗干净后, 经 $0.05 \%$ 杀真菌剂进行表面灭菌。灭过菌的具有 芽眼的菊芋块茎切片播于置于周转箱中装有石英砂 且下部有孔的塑料盆钵中, 周转箱存有一定深度的 培养液, 隔天换培养液, 每次都充分淋洗石英砂, 以 保证培养条件的均一性, 自然光照。待块茎萌发后 用 $1 / 2$ Hoagland 营养液 (含 $\mathrm{KNO}_{3} 2.525 \times 10^{-1} \mathrm{~g}$ ・ $\mathrm{L}^{-1}, \mathrm{Ca}\left(\mathrm{NO}_{3}\right)_{2} 4.100 \times 10^{-1} \mathrm{~g} \bullet \mathrm{L}^{-1}, \mathrm{KH}_{2} \mathrm{PO}_{4} 6.800 \times$ $10^{-2} \mathrm{~g} \bullet \mathrm{L}^{-1}, \mathrm{MgSO}_{4} 1.2 \times 10^{-1} \mathrm{~g} \bullet \mathrm{L}^{-1}, \mathrm{FeSO}_{4} \cdot 7 \mathrm{H}_{2} \mathrm{O}$ $5.57 \times 10^{-3} \mathrm{~g} \cdot \mathrm{L}^{-1}, \mathrm{H}_{3} \mathrm{BO}_{3} 2.86 \times 10^{-3} \mathrm{~g} \cdot \mathrm{L}^{-1}$, $\mathrm{MnCl}_{2} \cdot 7 \mathrm{H}_{2} \mathrm{O} 1.81 \times 10^{-3} \mathrm{~g} \bullet \mathrm{L}^{-1}, \mathrm{ZnSO}_{4} \cdot 7 \mathrm{H}_{2} \mathrm{O} 2.2 \times$ $10^{-4} \mathrm{~g} \cdot \mathrm{L}^{-1}, \mathrm{CuSO}_{4} \cdot 7 \mathrm{H}_{2} \mathrm{O} 0.8 \times 10^{-4} \mathrm{~g} \cdot \mathrm{L}^{-1}$, $\left.\left(\mathrm{NH}_{4}\right)_{2} \mathrm{MoO}_{4} \quad 0.9 \times 10^{-4} \mathrm{~g} \cdot \mathrm{L}^{-1}\right)$ 浇灌。设 $\mathrm{CK}($ 去离 子水)、 $10 \%$ 海水 ( $100 \%$ 莱州湾海水基本理化性质见 表 1)、10\%海水 $+8 \mathrm{mmol} \cdot \mathrm{L}^{-1} \mathrm{P} 、 25 \%$ 海水和 $25 \%$ 海 水 $+8 \mathrm{mmol} \cdot \mathrm{L}^{-1} \mathrm{P} 5$ 个处理, 分别以 $\mathrm{S}_{0} 、 \mathrm{~S}_{1} 、 \mathrm{~S}_{1} \mathrm{P} 、 \mathrm{~S}_{2}$ 和 $\mathrm{S}_{2} \mathrm{P}$ 表示, 磷素以 $\mathrm{NaH}_{2} \mathrm{PO}_{4}$ 提供, 分别用所需固体海 水 (天然海水蒸发所得) 和 $\mathrm{NaH}_{2} \mathrm{PO}_{4}$ 溶于 $1 / 2$ Hoagland 营养液以配制所需的各处理培养液。菊芋 幼苗长至 3 叶期时, 进行疏苗, 每盆保留 1 株, 至 6 叶完全展开时进行处理, 分别在处理后第四、八和十 二天采样进行分析, 各处理每次采样分析重复 6 次。

表 1 莱州湾海水基本离子组成

Table 1 Basic ion contents of Laizhou bay seawater

\begin{tabular}{|c|c|c|c|c|c|c|c|c|c|}
\hline \multirow{2}{*}{$\mathrm{pH}$} & \multicolumn{4}{|c|}{ 阴离子 $\operatorname{Anion}\left(\mathrm{g}^{\bullet} \mathrm{L}^{-1}\right)$} & \multicolumn{4}{|c|}{ 阳离子 Cation $\left(\mathrm{g} \bullet \mathrm{L}^{-1}\right)$} & \multirow{2}{*}{$\begin{array}{c}\text { 含盐量 } \\
\text { Salinity }\left(g \bullet L^{-1}\right)\end{array}$} \\
\hline & $\mathrm{CO}_{3}{ }^{2-}$ & $\mathrm{HCO}_{3}^{-}$ & $\mathrm{Cl}^{-}$ & $\mathrm{SO}_{4}^{2-}$ & $\mathrm{Ca}^{2+}$ & $\mathrm{Mg}^{2+}$ & $\mathrm{K}^{+}$ & $\mathrm{Na}^{+}$ & \\
\hline 8.30 & - & 0.132 & 17.52 & 3.867 & 0.785 & 1.027 & 0.596 & 9.480 & 33.33 \\
\hline
\end{tabular}

\section{2 鲜重和干重的测定}

将菊芋幼苗从塑料盆中取出, 用去离子水冲去 砂粒, 再用吸水纸吸干称地上部、地下部及全株鲜 重, 经 $110{ }^{\circ} \mathrm{C}$ 杀青 $10 \mathrm{~min}$ 后于 $60{ }^{\circ} \mathrm{C}$ 烘干至恒重。

1.3 测定方法
参照 Irigoyen 等 (1992) 的方法测定可溶性糖 ( SS) 和游离脯氨酸 (Pro)。

参照 Dhindsa 等 (1981) 的方法测定超氧化物岐 化酶 (SOD)活性, 参照 Chance 和 Maehly (1955) 的方 法测定过氧化氢酶( CAT) 活性和过氧化物酶 (POD) 
活性, 参照 Heath 和 Packer(1968) 方法测定丙二醛 (MDA)。

\section{参照 Liu 等(1985)方法测定质膜相对透性。}

1.4 无机离子含量的测定

取一定量植株地上部和地下部烘干磨细过 30 目篮。用火焰原子吸收分光光度计测定 $\mathrm{Na}^{+}$和 $\mathrm{K}^{+}$, 用原子吸收分光光度计测定 $\mathrm{Ca}^{2+}$ 和 $\mathrm{Mg}^{2+}$ ( Hunt, 1982)。滴定法测定 $\mathrm{Cl}^{-}$含量(於丙军等,2001)。

\section{2 结果与分析}

2.1 不同处理对菊芋幼苗生长发育的影响

随着时间延长, $\mathrm{S}_{1}$ 处理下, 菊芋幼苗地上部和 根鲜重均增加, 且与对照没有显著差异, $S_{2}$ 处理下 生长速率较对照低, 在第十二天时地上部和根鲜重 只有对照的 $82.3 \%$ 和 $86.9 \%$, 地上部和根干重情况 与鲜重情况类似。在 $\mathrm{S}_{1} \mathrm{P}$ 和 $\mathrm{S}_{2} \mathrm{P}$ 处理下,地上部和 根鲜重分别比 $S_{1}$ 和 $S_{2}$ 处理下高, 在第十二天时比
$\mathrm{S}_{1}$ 和 $\mathrm{S}_{2}$ 处理下高 $1.6 \% 、 3.2 \%$ 和 $3.1 \% 、 5.7 \%$, 说明 海水浓度越高, 磷素效应越大, 地上部和根干重情况 与鲜重情况类似 (表 2)。

2.2 不同处理对菊芋幼苗叶片脯氨酸及可溶性糖 含量的影响

如表 3 所示, 随着时间延长, 海水处理下菊芋幼 苗叶片脯氨酸含量先增加后减少, 在 $\mathrm{S}_{1}$ 和 $\mathrm{S}_{2}$ 处理 下, 脯氨酸含量较对照显著增加, 在第八天时分别是 对照的 1.89 和 31.35 倍, 在 $\mathrm{S}_{1} \mathrm{P}$ 和 $\mathrm{S}_{2} \mathrm{P}$ 处理下, 脯氨 酸含量分别比 $\mathrm{S}_{1}$ 和 $\mathrm{S}_{2}$ 处理下高, 在第八天时分别 比 $\mathrm{S}_{1}$ 和 $\mathrm{S}_{2}$ 处理下高 $145.7 \%$ 和 $53.3 \%$ 。

同样如表 3 所示, 随着时间延长, 可溶性糖含量 在 $\mathrm{S}_{0}$ 处理下增加后相对稳定, 而在 $\mathrm{S}_{1}$ 和 $\mathrm{S}_{2}$ 处理下 先增加后减少, 较对照显著增加, 在第八天时分别是 对照的 1.90 和 2.49 倍, 在 $\mathrm{S}_{1} \mathrm{P}$ 和 $\mathrm{S}_{2} \mathrm{P}$ 处理下, 可溶 性糖含量分别比 $\mathrm{S}_{1}$ 和 $\mathrm{S}_{2}$ 处理高, 在第八天时分别 比 $S_{1}$ 和 $S_{2}$ 处理高 $77.6 \%$ 和 $53.0 \%$ 。

表 2 不同处理对菊芋幼苗地上部和根鲜重及干重的影响

Table 2 Effects of different treatment on biomass fresh weight and dry weight of shoot and root of Helianthus tuberosus seedlings

\begin{tabular}{|c|c|c|c|c|c|c|c|c|c|c|c|}
\hline \multirow[b]{2}{*}{$\begin{array}{c}\text { 处理 } \\
\text { Treatment }\end{array}$} & \multirow[b]{2}{*}{$\begin{array}{l}\text { 天数 } \\
\text { Days }\end{array}$} & \multicolumn{2}{|c|}{$\mathrm{S}_{0}$} & \multicolumn{2}{|c|}{$\mathrm{S}_{1}$} & \multicolumn{2}{|c|}{$\mathrm{S}_{1} \mathrm{P}$} & \multicolumn{2}{|c|}{$\mathrm{S}_{2}$} & \multicolumn{2}{|c|}{$\mathrm{S}_{2} \mathrm{P}$} \\
\hline & & $\begin{array}{l}\text { 地上部 } \\
\text { Shoot }\end{array}$ & $\begin{array}{c}\text { 根 } \\
\text { Root }\end{array}$ & $\begin{array}{c}\text { 地上部 } \\
\text { Shoot }\end{array}$ & $\begin{array}{c}\text { 根 } \\
\text { Root }\end{array}$ & $\begin{array}{c}\text { 地上部 } \\
\text { Shoot }\end{array}$ & $\begin{array}{c}\text { 根 } \\
\text { Root }\end{array}$ & $\begin{array}{l}\text { 地上部 } \\
\text { Shoot }\end{array}$ & $\begin{array}{c}\text { 根 } \\
\text { Root }\end{array}$ & $\begin{array}{c}\text { 地上部 } \\
\text { Shoot }\end{array}$ & $\begin{array}{c}\text { 根 } \\
\text { Root }\end{array}$ \\
\hline 鲜重 & 4 & $3.48^{\mathrm{b}}$ & $3.30^{c}$ & $3.56^{\mathrm{b}}$ & $3.54^{\mathrm{b}}$ & $3.84^{\mathrm{a}}$ & $3.67^{\mathrm{a}}$ & $3.36^{c}$ & $3.65^{\mathrm{a}}$ & $3.48^{\mathrm{b}}$ & $3.73^{\mathrm{a}}$ \\
\hline Fresh weight & 8 & $4.26^{\mathrm{cd}}$ & $4.35^{\mathrm{cd}}$ & $4.58^{\mathrm{b}}$ & $4.68^{\mathrm{b}}$ & $4.67^{\mathrm{a}}$ & $4.85^{\mathrm{a}}$ & $4.18^{\mathrm{d}}$ & $4.23^{\mathrm{d}}$ & $4.32^{\mathrm{c}}$ & $4.46^{\mathrm{c}}$ \\
\hline$\left(\mathrm{g}^{\bullet}\right.$ plant $\left.^{-1}\right)$ & 12 & $5.58^{\mathrm{a}}$ & $5.03^{\mathrm{ab}}$ & $5.59^{\mathrm{a}}$ & $4.96^{\mathrm{b}}$ & $5.68^{\mathrm{a}}$ & $5.12^{\mathrm{a}}$ & $4.59^{\mathrm{c}}$ & $4.37^{\mathrm{d}}$ & $4.73^{\mathrm{b}}$ & $4.62^{\mathrm{c}}$ \\
\hline 干重 & 4 & $0.42^{\mathrm{b}}$ & $0.19^{\mathrm{c}}$ & $0.43^{\mathrm{ab}}$ & $0.20^{\mathrm{bc}}$ & $0.45^{\mathrm{a}}$ & $0.21^{\mathrm{ab}}$ & $0.38^{\mathrm{d}}$ & $0.21^{\mathrm{a}}$ & $0.39^{\mathrm{cd}}$ & $0.22^{\mathrm{a}}$ \\
\hline Dry weight & 8 & $0.49^{\mathrm{b}}$ & $0.25^{\mathrm{b}}$ & $0.53^{\mathrm{a}}$ & $0.26^{\mathrm{b}}$ & $0.54^{\mathrm{a}}$ & $0.28^{\mathrm{a}}$ & $0.46^{\mathrm{c}}$ & $0.25^{\mathrm{b}}$ & $0.48^{\mathrm{bc}}$ & $0.27^{\mathrm{ab}}$ \\
\hline$\left(\mathrm{g} \bullet\right.$ plant $\left.^{-1}\right)$ & 12 & $0.59^{\mathrm{a}}$ & $0.33^{\mathrm{a}}$ & $0.58^{\mathrm{a}}$ & $0.32^{\mathrm{a}}$ & $0.59^{\mathrm{a}}$ & $0.33^{\mathrm{a}}$ & $0.53^{\mathrm{b}}$ & $0.30^{\mathrm{b}}$ & $0.55^{\mathrm{b}}$ & $0.32^{\mathrm{a}}$ \\
\hline
\end{tabular}

表中同项同行数据后相同字母表示在 $p=0.05$ 水平上无显著差异 Data followed by same letters are not significantly different at $p=0.05$ level in a line

表 3 不同处理对菊芋幼苗叶片脯氨酸及可溶性糖含量的影响

Table 3 Effects of different treatment on proline and soluble-suger content in leaf of Helianthus tuberosus seedlings

\begin{tabular}{|c|c|c|c|c|c|c|}
\hline 处理 Treatment & 天数 Days & $\mathrm{S}_{0}$ & $\mathrm{~S}_{1}$ & $\mathrm{~S}_{1} \mathrm{P}$ & $\mathrm{S}_{2}$ & $\mathrm{~S}_{2} \mathrm{P}$ \\
\hline $\begin{array}{l}\text { 脯氨酸 } \\
\end{array}$ & 4 & $17.12^{\mathrm{cd}}$ & $15.92^{\mathrm{d}}$ & $24.21^{\mathrm{b}}$ & $17.38^{\mathrm{c}}$ & $41.72^{\mathrm{a}}$ \\
\hline \multirow[t]{2}{*}{ Proline $\left(\mu \mathrm{g} \bullet \mathrm{g}^{-1} \mathrm{FW}\right)$} & 8 & $8.97^{\mathrm{e}}$ & $16.95^{\mathrm{d}}$ & $41.64^{c}$ & $281.23^{b}$ & $431.08^{\mathrm{a}}$ \\
\hline & 12 & $5.20^{\mathrm{e}}$ & $14.37^{\mathrm{d}}$ & $23.73^{\mathrm{c}}$ & $118.91^{\mathrm{b}}$ & $233.68^{\mathrm{a}}$ \\
\hline \multirow{3}{*}{$\begin{array}{c}\text { 可溶性糖 } \\
\text { Soluble-suger }(\%)\end{array}$} & 4 & $0.55^{\mathrm{d}}$ & $0.74^{\mathrm{c}}$ & $0.89^{\mathrm{bc}}$ & $0.98^{\mathrm{b}}$ & $1.17^{\mathrm{a}}$ \\
\hline & 8 & $0.87^{\mathrm{e}}$ & $1.65^{\mathrm{d}}$ & $2.93^{\mathrm{b}}$ & $2.17^{\mathrm{c}}$ & $3.32^{\mathrm{a}}$ \\
\hline & 12 & $0.90^{\mathrm{e}}$ & $1.01^{\mathrm{de}}$ & $1.16^{\mathrm{cd}}$ & $1.57^{\mathrm{b}}$ & $1.91^{\mathrm{a}}$ \\
\hline
\end{tabular}
line

表中同项同行数据后相同字母表示在 $p=0.05$ 水平上无显著差异 Data followed by same letters are not significantly different at $p=0.05$ level in a

2.3 不同处理对菊芋幼苗叶片 SOD、POD 及 CAT 活 性的影响

如表 4 所示, 随着时间延长, SOD 活性在 $\mathrm{S}_{0}$ 处 理下相对稳定, 而在 $S_{1}$ 和 $S_{2}$ 处理下增加, 第十二天 时较第四天时分别高 $48.1 \%$ 和 $61.6 \%$, 同时较对照
增大, 在第十二天时分别是对照的 1.17 和 1.86 倍, 在 $\mathrm{S}_{1} \mathrm{P}$ 和 $\mathrm{S}_{2} \mathrm{P}$ 处理下, $\mathrm{SOD}$ 活性分别比 $\mathrm{S}_{1}$ 和 $\mathrm{S}_{2}$ 处理 下高, 在第十二天时分别比 $\mathrm{S}_{1}$ 和 $\mathrm{S}_{2}$ 处理下 SOD 活 性高 $14.8 \%$ 和 $30.0 \%$ 。

同样如表 4 所示, 随着时间延长, POD 活性在 $\mathrm{S}_{0}$ 
处理下先增加后相对稳定, 而在 $S_{1} 、 S_{1} P 、 S_{2}$ 和 $S_{2} P$ 处 理先增加后降低, 同时较对照显著增加, 在第八天时 分别是对照的 $1.32 、 1.63 、 1.29$ 和 1.42 倍, 在 $\mathrm{S}_{1} \mathrm{P}$ 和 $\mathrm{S}_{2} \mathrm{P}$ 处理下, POD 活性分别比 $\mathrm{S}_{1}$ 和 $\mathrm{S}_{2}$ 处理下高, 在第十二天时分别比 $\mathrm{S}_{1}$ 和 $\mathrm{S}_{2}$ 处理下 POD 活性高 $26.7 \%$ 和 $12.0 \%$ 。

随着时间延长, CAT 活性在 $\mathrm{S}_{0} 、 \mathrm{~S}_{1} 、 \mathrm{~S}_{1} \mathrm{P} 、 \mathrm{~S}_{2}$ 和 $\mathrm{S}_{2} \mathrm{P}$ 处理下均增加, 同时在 $S_{1} 、 S_{1} P 、 S_{2}$ 和 $S_{2} P$ 处理下较对 照差异显著, 在第十二天时分别是对照的 1.55 、 2.08、1.99 和 2.55 倍, 在 $\mathrm{S}_{1} \mathrm{P}$ 和 $\mathrm{S}_{2} \mathrm{P}$ 处理下, CAT 活 性分别比在 $S_{1}$ 和 $S_{2}$ 处理下高, 在第十二天时分别 比 $\mathrm{S}_{1}$ 和 $\mathrm{S}_{2}$ 处理下高 $33.9 \%$ 和 $28.4 \%$ (表 4)。

2.4 不同处理对菊芋幼苗叶片 MDA 含量和膜透性 的影响

随着时间延长, MDA 含量在 $\mathrm{S}_{0} 、 \mathrm{~S}_{1} 、 \mathrm{~S}_{1} \mathrm{P} 、 \mathrm{~S}_{2}$ 和
$\mathrm{S}_{2} \mathrm{P}$ 处理下均先增加后降低, 在 $\mathrm{S}_{1}$ 和 $\mathrm{S}_{1} \mathrm{P}$ 处理下较 对照差异不大, 而在 $\mathrm{S}_{2}$ 和 $\mathrm{S}_{2} \mathrm{P}$ 处理下较对照差异显 著, 在第八天时分别是对照的 1.59 和 1.40 倍, 在 $\mathrm{S}_{1} \mathrm{P}$ 和 $\mathrm{S}_{2} \mathrm{P}$ 处理下, MDA 含量分别比 $\mathrm{S}_{1}$ 和 $\mathrm{S}_{2}$ 处理 低, 在第十二天时分别比 $\mathrm{S}_{1}$ 和 $\mathrm{S}_{2}$ 处理低 $31.0 \%$ 和 $23.4 \%$ (表 5)。

从表 5 可知, 随着时间延长, 膜透性在 $\mathrm{S}_{0}$ 处理 下增加后相对稳定, 而在 $\mathrm{S}_{1}$ 和 $\mathrm{S}_{2}$ 处理下增大, 第十 二天时较第四天时分别高 $8.7 \%$ 和 $28.6 \%$, 同时较 对照显著增加, 在第十二天时分别是对照的 1.05 和 1.49 倍, 说明海水浓度越高, 膜透性增加幅度越大, 在 $\mathrm{S}_{1} \mathrm{P}$ 和 $\mathrm{S}_{2} \mathrm{P}$ 处理下, 膜透性分别比 $\mathrm{S}_{1}$ 和 $\mathrm{S}_{2}$ 处理下 低, 在第八天时分别比 $S_{1}$ 和 $S_{2}$ 处理下膜透性低 $3.0 \%$ 和 $9.5 \%$ 。

表 4 不同处理对菊芋幼苗叶片 SOD, POD 和 CAT 活性的影响

Table 4 Effects of different treatment on SOD, POD and CAT activity in leaf of Helianthus tuberosus seedlings

\begin{tabular}{|c|c|c|c|c|c|c|}
\hline 处理 Treatment & 天数 Days & $\mathrm{S}_{0}$ & $\mathrm{~S}_{1}$ & $\mathrm{~S}_{1} \mathrm{P}$ & $\mathrm{S}_{2}$ & $\mathrm{~S}_{2} \mathrm{P}$ \\
\hline \multirow{3}{*}{$\begin{array}{c}\text { SOD } \\
\left(\mathrm{U} \cdot \mathrm{mg}^{-1} \text { protein }\right)\end{array}$} & 4 & $3.88^{\mathrm{b}}$ & $3.37^{\mathrm{d}}$ & $3.68^{c}$ & $3.78^{\mathrm{bc}}$ & $4.26^{\mathrm{a}}$ \\
\hline & 8 & $3.98^{\mathrm{d}}$ & $3.62^{\mathrm{e}}$ & $4.15^{\mathrm{cd}}$ & $5.05^{\mathrm{b}}$ & $5.66^{\mathrm{a}}$ \\
\hline & 12 & $4.28^{\mathrm{e}}$ & $4.99^{\mathrm{d}}$ & $5.73^{\mathrm{c}}$ & $6.11^{\mathrm{b}}$ & $7.94^{\mathrm{a}}$ \\
\hline \multirow{3}{*}{$\begin{array}{c}\text { POD } \\
\left(\mathrm{mg}^{-1} \text { protein } \cdot \min ^{-1}\right)\end{array}$} & 4 & $0.023^{\mathrm{d}}$ & $0.046^{\mathrm{c}}$ & $0.058^{\mathrm{b}}$ & $0.062^{\mathrm{b}}$ & $0.088^{\mathrm{a}}$ \\
\hline & 8 & $0.304^{\mathrm{d}}$ & $0.400^{\mathrm{c}}$ & $0.496^{\mathrm{a}}$ & $0.393^{c}$ & $0.432^{\mathrm{b}}$ \\
\hline & 12 & $0.316^{\mathrm{d}}$ & $0.337^{\mathrm{d}}$ & $0.427^{\mathrm{a}}$ & $0.359^{c}$ & $0.402^{\mathrm{b}}$ \\
\hline \multirow{3}{*}{$\begin{array}{c}\text { CAT } \\
\left(\mathrm{mg}^{-1} \text { protein } \cdot \min ^{-1}\right)\end{array}$} & 4 & $0.080^{\mathrm{b}}$ & $0.062^{\mathrm{c}}$ & $0.084^{b}$ & $0.075^{\text {be }}$ & $0.147^{\mathrm{a}}$ \\
\hline & 8 & $0.152^{\mathrm{c}}$ & $0.116^{\mathrm{d}}$ & $0.186^{\mathrm{b}}$ & $0.198^{\mathrm{b}}$ & $0.262^{\mathrm{a}}$ \\
\hline & 12 & $0.236^{\mathrm{d}}$ & $0.366^{\mathrm{c}}$ & $0.490^{\mathrm{b}}$ & $0.469^{\mathrm{b}}$ & $0.602^{\mathrm{a}}$ \\
\hline
\end{tabular}

表中同项同行数据后相同字母表示在 $p=0.05$ 水平上无显著差异 Data followed by same letters are not significantly different at $p=0.05$ level in a line

表 5 不同处理对菊芋幼苗叶片 MDA 含量和膜透性的影响

Table 5 Effects of different treatment on MDA content and electrolytic leakage percentage ( ELP) in leaf of Helianthus tuberosus seedlings

\begin{tabular}{|c|c|c|c|c|c|c|}
\hline 处理 Treatment & 天数 Days & $\mathrm{S}_{0}$ & $\mathrm{~S}_{1}$ & $\mathrm{~S}_{1} \mathrm{P}$ & $\mathrm{S}_{2}$ & $\mathrm{~S}_{2} \mathrm{P}$ \\
\hline \multirow{3}{*}{$\begin{array}{c}\mathrm{MDA} \\
\left(\mathrm{nmol} \cdot \mathrm{g}^{-1} \mathrm{FW}\right)\end{array}$} & 4 & $4.72^{\mathrm{c}}$ & $4.82^{\mathrm{b}}$ & $4.84^{\mathrm{b}}$ & $5.38^{\mathrm{a}}$ & $4.85^{\mathrm{b}}$ \\
\hline & 8 & $5.77^{\mathrm{c}}$ & $5.38^{\mathrm{de}}$ & $5.15^{\mathrm{e}}$ & $9.19^{\mathrm{a}}$ & $8.10^{\mathrm{b}}$ \\
\hline & 12 & $3.70^{\mathrm{de}}$ & $4.91^{\mathrm{c}}$ & $3.39^{\mathrm{e}}$ & $7.53^{\mathrm{a}}$ & $5.77^{\mathrm{b}}$ \\
\hline \multirow{3}{*}{$\begin{array}{l}\text { 膜透性 } \\
\operatorname{ELP}(\%)\end{array}$} & 4 & $4.99^{\mathrm{bc}}$ & $5.03^{\mathrm{b}}$ & $4.87^{\mathrm{cd}}$ & $5.24^{\mathrm{a}}$ & $4.74^{\mathrm{d}}$ \\
\hline & 8 & $5.08^{\mathrm{d}}$ & $5.31^{\mathrm{bc}}$ & $5.15^{\mathrm{cd}}$ & $5.88^{\mathrm{a}}$ & $5.32^{\mathrm{b}}$ \\
\hline & 12 & $5.20^{\mathrm{d}}$ & $5.47^{\mathrm{c}}$ & $5.17^{\mathrm{d}}$ & $6.74^{\mathrm{a}}$ & $6.03^{\mathrm{b}}$ \\
\hline
\end{tabular}
line

2.5 不同处理对菊芋幼苗离子吸收分布的影响 如表 6 所示, 随着时间延长, 地上部和根部 $\mathrm{K}^{+}$ 含量在各处理下先降低后相对稳定, 在 $\mathrm{S}_{1} \mathrm{P} 、 \mathrm{~S}_{2}$ 和 $\mathrm{S}_{2} \mathrm{P}$ 处理下地上部 $\mathrm{K}^{+}$含量较对照显著增加, 在第四 天时分别是对照的 $1.15 、 1.17$ 和 1.30 倍, 而根部 $\mathrm{K}^{+}$含量较对照差异更显著, 在第四天时分别是对照
的 1.67、1.42 和 1.53 倍。在 $\mathrm{S}_{1} \mathrm{P}$ 和 $\mathrm{S}_{2} \mathrm{P}$ 处理下, 地 上部和根部 $\mathrm{K}^{+}$含量分别比 $\mathrm{S}_{1}$ 和 $\mathrm{S}_{2}$ 处理增加, 说明 在海水胁迫下磷素有利于 $\mathrm{K}^{+}$的吸收分布。

同样如表 6 所示, 随着时间延长, 在 $\mathrm{S}_{0}$ 处理下 地上部和根部 $\mathrm{Na}^{+}$含量变化不大, 而在 $\mathrm{S}_{1} 、 \mathrm{~S}_{1} \mathrm{P} 、 \mathrm{~S}_{2}$ 和 $\mathrm{S}_{2} \mathrm{P}$ 处理下均增加, 且海水浓度越大增加幅度越大, 
在第十二天时分别是对照的 7.86、6.29、18.43、 14.86倍和 $5.29 、 4.18 、 7.11 、 4.82$ 倍。在 $\mathrm{S}_{1} \mathrm{P}$ 和 $\mathrm{S}_{2} \mathrm{P}$ 处理下, 地上部和根部 $\mathrm{Na}^{+}$含量分别比 $\mathrm{S}_{1}$ 和 $\mathrm{S}_{2}$ 处理 降低, 在第十二天时分别比 $\mathrm{S}_{1}$ 和 $\mathrm{S}_{2}$ 处理低 $20.0 \%$ 、 $19.4 \%$ 和 $20.9 \% 、 32.2 \%$ 。

随着时间延长, 地上部和根部 $\mathrm{Ca}^{2+}$ 含量在 $\mathrm{S}_{0}$ 处 理下相对稳定, 在 $S_{1} 、 S_{1} P 、 S_{2}$ 和 $S_{2} P$ 处理下 $\mathrm{Ca}^{2+}$ 含量 增加, 较对照差异明显, 在第十二天时分别是对照的 $1.28 、 1.50 、 1.57 、 2.14$ 倍和 $1.20 、 1.33 、 1.27 、 1.53$ 倍。在 $\mathrm{S}_{1} \mathrm{P}$ 和 $\mathrm{S}_{2} \mathrm{P}$ 处理下, 地上部和根部 $\mathrm{Ca}^{2+}$ 含量 分别比 $\mathrm{S}_{1}$ 和 $\mathrm{S}_{2}$ 处理增加, 在第十二天时分别比 $\mathrm{S}_{1}$ 和 $\mathrm{S}_{2}$ 处理高 $16.7 \% 、 36.4 \%$ 和 $11.1 \% 、 21.1 \%$, 说明 在海水胁迫下磷素有利于 $\mathrm{Ca}^{2+}$ 的吸收分布(表 6)。

同样由表 6 可知, 随着时间延长, 地上部和根部 $\mathrm{Mg}^{2+}$ 含量在 $\mathrm{S}_{0} 、 \mathrm{~S}_{1} 、 \mathrm{~S}_{1} \mathrm{P} 、 \mathrm{~S}_{2}$ 和 $\mathrm{S}_{2} \mathrm{P}$ 处理下 $\mathrm{Mg}^{2+}$ 含量
增加, 且 $S_{1} 、 S_{1} P 、 S_{2}$ 和 $S_{2} P$ 处理下 $\mathrm{Mg}^{2+}$ 含量较对照 差异明显, 在第十二天时分别是对照的 $1.10 、 1.25$ 、 $2.05 、 2.35$ 倍和 $1.17 、 1.29 、 1.40 、 1.54$ 倍。在 $\mathrm{S}_{1} \mathrm{P}$ 和 $\mathrm{S}_{2} \mathrm{P}$ 处理下, 地上部和根部 $\mathrm{Mg}^{2}+$ 含量分别比 $\mathrm{S}_{1}$ 和 $\mathrm{S}_{2}$ 处理增加, 在第十二天时分别比 $\mathrm{S}_{1}$ 和 $\mathrm{S}_{2}$ 处理高 $13.6 \% 、 14.6 \%$ 和 $9.8 \% 、 10.2 \%$, 说明在海水胁迫下 磷素也有利于 $\mathrm{Mg}^{2+}$ 的吸收分布。

同地上部和根部 $\mathrm{Na}^{+}$含量变化相似, 随着时间 延长, 在 $\mathrm{S}_{0}$ 处理下地上部和根部 $\mathrm{Cl}^{-}$含量变化不 大, 而在 $S_{1} 、 S_{1} P 、 S_{2}$ 和 $S_{2} P$ 处理下均增加, 且海水浓 度越大增加幅度越大, 在第十二天时分别是对照的 $4.93 、 3.78 、 7.63 、 6.11$ 倍和 $6.65 、 5.10 、 7.29 、 5.77$ 倍。在 $\mathrm{S}_{1} \mathrm{P}$ 和 $\mathrm{S}_{2} \mathrm{P}$ 处理下, 地上部和根部 $\mathrm{Cl}^{-}$含量分 别比 $S_{1}$ 和 $S_{2}$ 处理降低, 在第十二天时分别比 $S_{1}$ 和 $\mathrm{S}_{2}$ 处理低 $23.3 \% 、 19.9 \%$ 和 $23.3 \% 、 20.8 \%$ (表 6)。

表 6 不同处理对菊芋幼苗地上部和根 $\mathrm{K}^{+} 、 \mathrm{Na}^{+} 、 \mathrm{Ca}^{2+} 、 \mathrm{Mg}^{2+}$ 和 $\mathrm{Cl}^{-}$含量的影响

Table 6 Effects of different treatment on $\mathrm{K}^{+} 、 \mathrm{Na}^{+} 、 \mathrm{Ca}^{2+} 、 \mathrm{Mg}^{2+}$ and $\mathrm{Cl}^{-}$content in shoot and root of Helianthus tuberosus seedlings

\begin{tabular}{|c|c|c|c|c|c|c|c|c|c|c|c|}
\hline \multirow{2}{*}{$\begin{array}{c}\text { 处理 } \\
\text { Treatment }\end{array}$} & \multirow{2}{*}{$\begin{array}{l}\text { 天数 } \\
\text { Days }\end{array}$} & \multicolumn{2}{|c|}{$\mathrm{S}_{0}$} & \multicolumn{2}{|c|}{$\mathrm{S}_{1}$} & \multicolumn{2}{|c|}{$\mathrm{S}_{1} \mathrm{P}$} & \multicolumn{2}{|c|}{$\mathrm{S}_{2}$} & \multicolumn{2}{|c|}{$\mathrm{S}_{2} \mathrm{P}$} \\
\hline & & $\begin{array}{c}\text { 地上部 } \\
\text { Shoot }\end{array}$ & $\begin{array}{c}\text { 根 } \\
\text { Root }\end{array}$ & $\begin{array}{c}\text { 地上部 } \\
\text { Shoot }\end{array}$ & $\begin{array}{c}\text { 根 } \\
\text { Root }\end{array}$ & $\begin{array}{c}\text { 地上部 } \\
\text { Shoot }\end{array}$ & $\begin{array}{c}\text { 根 } \\
\text { Root }\end{array}$ & $\begin{array}{c}\text { 地上部 } \\
\text { Shoot }\end{array}$ & $\begin{array}{c}\text { 根 } \\
\text { Root }\end{array}$ & $\begin{array}{c}\text { 地上部 } \\
\text { Shoot }\end{array}$ & $\begin{array}{c}\text { 根 } \\
\text { Root }\end{array}$ \\
\hline $\mathrm{K}^{+}$ & 4 & $1.24^{\mathrm{c}}$ & $1.16^{\mathrm{d}}$ & $1.44^{\mathrm{b}}$ & $1.61^{\mathrm{c}}$ & $1.43^{\mathrm{b}}$ & $1.94^{\mathrm{a}}$ & $1.45^{\mathrm{b}}$ & $1.65^{\mathrm{c}}$ & $1.61^{\mathrm{a}}$ & $1.77^{\mathrm{b}}$ \\
\hline （ $\mathrm{mmol}^{\bullet}$ & 8 & $1.12^{\mathrm{c}}$ & $1.29^{c}$ & $1.10^{\mathrm{c}}$ & $1.32^{\mathrm{c}}$ & $1.20^{\mathrm{b}}$ & $1.48^{\mathrm{b}}$ & $1.14^{c}$ & $1.45^{\mathrm{b}}$ & $1.38^{\mathrm{a}}$ & $1.64^{\mathrm{a}}$ \\
\hline $\left.\mathrm{g}^{-1} \mathrm{DW}\right)$ & 12 & $1.04^{\mathrm{c}}$ & $1.20^{\mathrm{c}}$ & $1.05^{\mathrm{c}}$ & $1.41^{\mathrm{b}}$ & $1.18^{\mathrm{b}}$ & $1.52^{\mathrm{a}}$ & $1.21^{\mathrm{b}}$ & $1.40^{\mathrm{b}}$ & $1.49^{\mathrm{a}}$ & $1.47^{\mathrm{ab}}$ \\
\hline $\mathrm{Na}^{+}$ & 4 & $0.05^{\mathrm{d}}$ & $0.16^{\mathrm{d}}$ & $0.17^{\mathrm{c}}$ & $0.73^{\mathrm{b}}$ & $0.16^{\mathrm{c}}$ & $0.52^{\mathrm{c}}$ & $0.71^{\mathrm{a}}$ & $1.07^{\mathrm{a}}$ & $0.53^{\mathrm{b}}$ & $0.79^{\mathrm{b}}$ \\
\hline$(\mathrm{mmol} \bullet$ & 8 & $0.06^{\mathrm{e}}$ & $0.31^{\mathrm{d}}$ & $0.41^{\mathrm{c}}$ & $1.13^{\mathrm{b}}$ & $0.28^{\mathrm{d}}$ & $0.91^{\mathrm{c}}$ & $1.05^{\mathrm{a}}$ & $1.32^{\mathrm{a}}$ & $0.61^{\mathrm{b}}$ & $1.05^{\mathrm{bc}}$ \\
\hline $\left.\mathrm{g}^{-1} \mathrm{DW}\right)$ & 12 & $0.07^{\mathrm{d}}$ & $0.28^{\mathrm{e}}$ & $0.55^{\mathrm{c}}$ & $1.48^{\mathrm{bc}}$ & $0.44^{\mathrm{c}}$ & $1.17^{\mathrm{d}}$ & $1.29^{\mathrm{a}}$ & $1.99^{\mathrm{a}}$ & $1.04^{\mathrm{b}}$ & $1.35^{\mathrm{c}}$ \\
\hline $\mathrm{Ca}^{2+}$ & 4 & $0.13^{\mathrm{b}}$ & $0.13^{c}$ & $0.14^{\mathrm{b}}$ & $0.14^{\mathrm{bc}}$ & $0.13^{\mathrm{b}}$ & $0.15^{\mathrm{b}}$ & $0.13^{\mathrm{b}}$ & $0.14^{\mathrm{bc}}$ & $0.17^{\mathrm{a}}$ & $0.18^{\mathrm{a}}$ \\
\hline$(\mathrm{mmol} \bullet$ & 8 & $0.13^{\mathrm{d}}$ & $0.12^{\mathrm{d}}$ & $0.15^{\mathrm{cd}}$ & $0.13^{\mathrm{d}}$ & $0.17^{\text {be }}$ & $0.17^{\mathrm{bc}}$ & $0.18^{\mathrm{b}}$ & $0.16^{\mathrm{c}}$ & $0.23^{\mathrm{a}}$ & $0.19^{\mathrm{a}}$ \\
\hline $\left.\mathrm{g}^{-1} \mathrm{DW}\right)$ & 12 & $0.14^{\mathrm{d}}$ & $0.15^{\mathrm{d}}$ & $0.18^{\mathrm{c}}$ & $0.18^{\mathrm{c}}$ & $0.21^{\mathrm{b}}$ & $0.20^{\mathrm{b}}$ & $0.22^{\mathrm{b}}$ & $0.19^{\mathrm{bc}}$ & $0.30^{\mathrm{a}}$ & $0.23^{\mathrm{a}}$ \\
\hline $\mathrm{Mg}^{2+}$ & 4 & $0.10^{\mathrm{e}}$ & $0.23^{\mathrm{d}}$ & $0.15^{\mathrm{d}}$ & $0.24^{\mathrm{d}}$ & $0.20^{c}$ & $0.33^{\mathrm{b}}$ & $0.23^{\mathrm{b}}$ & $0.27^{\mathrm{c}}$ & $0.26^{\mathrm{a}}$ & $0.37^{\mathrm{a}}$ \\
\hline$(\mathrm{mmol} \bullet$ & 8 & $0.18^{\mathrm{e}}$ & $0.31^{\mathrm{d}}$ & $0.19^{\mathrm{de}}$ & $0.33^{\mathrm{cd}}$ & $0.21^{\mathrm{cd}}$ & $0.39^{\mathrm{b}}$ & $0.27^{\mathrm{b}}$ & $0.37^{\mathrm{b}}$ & $0.32^{\mathrm{a}}$ & $0.46^{\mathrm{a}}$ \\
\hline $\left.\mathrm{g}^{-1} \mathrm{DW}\right)$ & 12 & $0.20^{\mathrm{d}}$ & $0.35^{\mathrm{e}}$ & $0.22^{\mathrm{cd}}$ & $0.41^{\mathrm{d}}$ & $0.25^{\mathrm{c}}$ & $0.45^{\mathrm{c}}$ & $0.41^{\mathrm{b}}$ & $0.49^{\mathrm{b}}$ & $0.47^{\mathrm{a}}$ & $0.54^{\mathrm{a}}$ \\
\hline $\mathrm{Cl}^{-}$ & 4 & $0.21^{\mathrm{e}}$ & $0.27^{\mathrm{e}}$ & $0.87^{\mathrm{c}}$ & $1.06^{\mathrm{cd}}$ & $0.74^{\mathrm{d}}$ & $0.96^{\mathrm{d}}$ & $1.19^{\mathrm{a}}$ & $1.66^{\mathrm{a}}$ & $1.01^{\mathrm{b}}$ & $1.29^{\mathrm{b}}$ \\
\hline （ $\mathrm{mmol} \bullet$ & 8 & $0.26^{\mathrm{e}}$ & $0.37^{\mathrm{e}}$ & $1.16^{\mathrm{c}}$ & $1.70^{\mathrm{bc}}$ & $0.88^{\mathrm{d}}$ & $1.26^{\mathrm{d}}$ & $1.93^{\mathrm{a}}$ & $2.02^{\mathrm{a}}$ & $1.53^{\mathrm{b}}$ & $1.54^{c}$ \\
\hline $\left.\mathrm{g}^{-1} \mathrm{DW}\right)$ & 12 & $0.27^{\mathrm{e}}$ & $0.31^{\mathrm{e}}$ & $1.33^{\mathrm{c}}$ & $2.06^{\mathrm{b}}$ & $1.02^{\mathrm{d}}$ & $1.58^{\mathrm{d}}$ & $2.06^{\mathrm{a}}$ & $2.26^{\mathrm{a}}$ & $1.65^{\mathrm{b}}$ & $1.79^{\mathrm{c}}$ \\
\hline
\end{tabular}

表中同项同行数据后相同字母表示在 $p=0.05$ 水平上无显著差异 Data followed by same letters are not significantly different at $p=0.05$ level in a line

\section{3 讨 论}

一般认为, 中性盐 $(\mathrm{NaCl})$ 胁迫的伤害作用主要 是通过离子本身的毒性效应及盐离子所致的渗透效 应和营养效应来完成的, 提高植物的矿质营养是农 作物高产和高抗逆性的重要前提和生理基础(彭长 连等,2000)。本试验表明: $10 \%$ 海水对菊芋幼苗生 长发育没有抑制作用，甚至有一定的促进作用，而 $25 \%$ 海水胁迫对菊芋幼苗形态发育上具有一定抑制
作用, 整体表现为抑制植株苗期组织和器官生长, 叶 面积缩小, 茎干和根系伸长受到抑制, 这可能是高浓 度盐分影响了细胞分裂和细胞延伸速率而引起的, 但增加磷素浓度后, 能显著缓解其抑制作用, 表现为 促进菊芋幼苗地上部和根部鲜重及干重的增加。

大量研究表明, 许多植物在盐胁迫下脯氨酸迅 速积累, 一般认为脯氨酸作为渗透调节剂对盐、干旱 等胁迫起到缓冲保护作用, 或者作为细胞质酶类和 膜的保护剂, 对酶类和膜的稳定性起一定作用, 
(Demir \& Kocacaliskan, 2001; Fedina et al.,2002), 可 溶性糖是很多非盐生植物的主要渗透调节剂, 它也 是合成别的有机溶质的炭架和能量来源 (张海燕和 赵可夫, 1998), 还可以在细胞内无机离子高时起保 护酶类的作用 (Yan et al., 1994)。本试验结果 (表 3)表明菊芋幼苗经短期休克性反应之后出现了对 $10 \%$ 和 $25 \%$ 海水的一定程度的适应, 而增加磷素浓 度后, 能显著增加菊芋幼苗叶片脯氨酸和可溶性糖 含量, 表明磷素能够诱导菊芋幼苗叶片内渗透物质 累积量增加, 从而增强对细胞膜和原生质胶体的稳 定性, 使处于逆境条件下的植物不易受到伤害。

在盐胁迫下, 细胞内高盐环境导致细胞严重缺 水,打破体内的氧化还原平衡, 从而诱导产生活性 氧, 如 $\mathrm{O}_{2}^{*} 、 \mathrm{H}_{2} \mathrm{O}_{2} 、 \cdot \mathrm{OH}$ 和 ${ }^{1} \mathrm{O}_{2}$ 等 (Tanaka et al., 1998; Tiwari et al ., 2002), SOD、POD 和 CAT 共同组成植物 体内一个有效的活性氧清除系统, 三者协调一致的 共同作用, 能有效清除植物体内的自由基和过氧化 物(任红旭等, 2001)。本试验结果表明: 随时间延 长, 各处理下菊芋幼苗叶片 SOD 和 CAT 活性都增 加, POD 活性在 $10 \%$ 和 $25 \%$ 海水处理下先增加后降 低, 增加磷素浓度后, 能显著增加菊芋幼苗叶片 SOD、POD 和 CAT 活性, 说明磷素能够增强活性氧清 除系统的活性,维持了植株体内保护酶较高的合成 速度, 从而清除植物体内的自由基和过氧化物, 起到 减少具毒性和高活性的・ $\mathrm{OH}$ 的形成, 有效阻止 $\mathrm{O}_{2}{ }^{-}$ 和 $\mathrm{H}_{2} \mathrm{O}_{2}$ 的积累, 限制这些自由基对膜脂过氧化的启 动, 缓解植物生理代谢紊乱。

MDA 含量的多少可以代表膜损伤程度的大小， 膜透性是直接反映膜伤害的程度, MDA 是间接表示 膜受损状况, 并兼有反馈作用(储玲等, 2004)。本试 验结果(表 5) 表明在 $10 \%$ 海水处理下, 菊芋幼苗叶 片质膜没有受到损伤, 而 $25 \%$ 海水处理下菊芋幼苗 叶片 MDA 含量在第十二天较第八天下降, 说明在菊 芋幼苗经短期休克性反应之后出现了对 $25 \%$ 海水 的一定程度的适应,而膜透性变化情况与 MDA 含量 变化趋势不一致,增加磷素浓度后, 降低了菊芋幼苗 叶片 MDA 含量和膜透性, 说明磷素能够缓解植物膜 受到损伤。

进入植物组织的无机离子也就是通常所说的盐 分离子, 是参与植物渗透调节的重要物质, 盐分离子 在盐生植物中是主要的细胞渗透调节溶质(林鹏和 王文卿, 1999)。随着海水浓度增加, 菊芋幼苗地上 部和根 $\mathrm{Na}^{+}$和 $\mathrm{Cl}^{-}$含量显著增加, 且随着时间延长, 不同浓度海水处理下地上部和根部 $\mathrm{Na}^{+}$和 $\mathrm{Cl}^{-}$含量
均增大, 增加磷素浓度后, 均能降低地上部和根 $\mathrm{Na}^{+}$ 和 $\mathrm{Cl}^{-}$含量, 而地上部和根部 $\mathrm{K}^{+} 、 \mathrm{Ca}^{2+}$ 和 $\mathrm{Mg}^{2+}$ 含量 较对照增加, 增加磷素浓度后, 均能增加 $\mathrm{K}^{+} 、 \mathrm{Ca}^{2+}$ 和 $\mathrm{Mg}^{2}+$ 含量, 说明磷素能够抑制菊芋对 $\mathrm{Na}^{+}$及 $\mathrm{Cl}^{-}$ 等有害离子的吸收, 促进菊芋对 $\mathrm{K}^{+} 、 \mathrm{Ca}^{2+}$ 和 $\mathrm{Mg}^{2+}$ 等 有益离子的吸收与运输。

由此可见, 磷素能够改善菊芋幼苗的营养状况, 同时能够增强其抗盐性。导致以上结果的原因可能 是磷调节海水处理下菊芋幼苗根系等细胞质膜 $\mathrm{H}^{+}$ATPase、液泡膜 $\mathrm{H}^{+}-$ATPase $、 \mathrm{H}^{+}$-PPase 活性, 促进光 合作用中的光合磷酸化过程, 产生大量的 ATP, 增强 质膜的稳定性和钙信号系统的正常发生和传递, 并 激活质膜和液泡膜上 $\mathrm{Na}^{+} / \mathrm{H}^{+}$逆向运输蛋白, 从而 加速了 $\mathrm{K}^{+}$的吸收、 $\mathrm{Na}^{+}$的排放及 $\mathrm{Na}^{+}$在液泡中的积 累, 提高了 $\mathrm{K}^{+}$的选择性吸收和运输, 促使盐分区域 化分配, 同时提高原生质胶体水合度和充水度以及 构成细胞膜磷脂结构, 并以此流动性来适应海水浓 度的变化, 进而增强细胞的抗逆性。从整体水平上 阐明磷对菊芋耐一定浓度海水的调节作用尚待进一 步深入研究。

\section{参 考 文 献}

Chance B, Maehly AC (1955). Assay of catalases and peroxidases. In: Colouick SP, Kaplan NO eds. Methods in Enzymology. Academic Press Publisher, New York, 746 - 755.

Chu L (储玲), Liu DY (刘登义), Wang YB (王友保) (2004). Effect of copper pollution on seedling growth and activate oxygen metabolism of Trifolium pratense. Chinese Journal of Applied Ecology (应用生态学报), 15, 119 - 122. (in Chinese with English abstract)

Demir Y, Kocacaliskan I (2001). Effects of NaCl and proline on polyphenol oxidase activity in bean seedings. Bilogia Plantarum, 44, $607-609$.

Dhindsa RS, Plumb-Dhindsa P, Thorpe TA (1981). Leafs enescence correlated with increased levels of membrane permeability and lipid peroxidation, and decreased levels of superoxide dismutase and catalase. Journal of Experimental Botany, 32, 93 101 .

Fedina IS, Georgieva K, Grigoreva I (2002). Light-dark changes in proline content of barley leaves under salt stress. Biologia Plantarum, 45, 59-63.

Heath RL, Packer L (1968). Photoperoxidation in isolated chloroplast. I. Kinetics and stoichemistry of fatty acid peroxidation. Archives of Biochemistry and Biophysics, 25, $189-198$.

Hunt J (1982) . Dilute hydrochloric acid extraction of plant material for routine cation anaslysis. Communication in Soil Science and Plant Analysis, 13, 49 - 55 .

Irigoyen, JJ, Emerich DW, Sanchez-Diaz M (1992). Water stress 
induced changes in concentrations of proline and total soluble sugars in nodulated alfalfa ( $M$. sativa) plants. Physiologia Plantarum, 84, 55-60.

Lin P (林鹏), Wang WQ (王文卿) (1999). Changes in the caloric values of Kandelia candel seedings under salt stress. Acta Phytoecologica Sinica (植物生态学报), 23, 466 - 470. (in Chinese with English abstract)

Liu HX (刘鸿先), Zeng SX (曾韶西), Wang YR (王以柔), Li P (李平), Chen DF (陈德峰), Guo JY (郭俊彦) (1985). The effect of low temperature on superoxide dismutase in various organelles of cucumber seedling cotyledon with different cold tolerance. Acta Phytophysiol Sinica (植物生理学报), 11, 48 57. (in Chinese with English abstract)

Liu ZP (刘兆普), Liu L (刘玲), Chen MD (陈铭达) (2003). Study on the irrigation systems in agriculture by seawater. Journal of natural resources (自然资源学报)，18,423-429. (in Chinese with English abstract)

Long XH (隆小华), Liu ZP (刘兆普), Chen MD (陈铭达) (2005) . Coupling effect of salt and fertilizer application on $\mathrm{He}$ lianthus tuberosus irrigated with seawater in semiarid region. Acta Pedologica Sinica (土壤学报), 42, 91 - 97. (in Chinese with English abstract)

Long XH (隆小华), Liu ZP (刘兆普), Liu L (刘玲)（2004）. Effects of different concentrations of seawater on growths, developments and absorption of $\mathrm{P}$ of Helianthus tuberosus seedlings. Bulletin of botanical research (植物研究), 24, 331-334. (in Chinese with English abstract)

Lu JL (陆景陵) (1994). Plant Nutrition (植物营养学). China Agricultural University Press, Beijing, 32-33. (in Chinese) Peng CL (彭长连), Lin ZF (林植芳), Ling GZ (林桂珠) (2000) . Photosynthesis and water use efficiency in wheat varieties differing in phosphate use efficiency. Acta Agronomica Sini$c a$ (作物学报) , 26, 543-548. (in Chinese with English abstract)

Ren HX (任红旭)，Chen X (陈雄)，Wang YF (王亚馥)
(2001). Changes in antioxidative enzymes and polyamines in wheat seedings with different drought resistance under drought and salt stress. Acta Phytoecologica Sinica (植物生态学报), 25, 709 - 715. (in Chinese with English abstract)

Shao J (邵晶)， Liu L (刘玲), Liu ZP (刘兆普), Zheng QS (郑 青松) (2005). Alleviatory effects of phosphorus on growth inhibition of seawater-stressed aloe seedlings. Scientia Agricultura Sinica (中国农业科学), 38,843-848. (in Chinese with English abstract)

Tanaka Y, Hibino T, Hayashi Y (1998). Salt tolerance of transgenetic rice overpression yeast mitochondrial $\mathrm{Mn}$-SOD in chloroplasts. Plant Science, 148, $131-138$.

Tiwari BS, Belenghi B, Levine A (2002). Oxidative stress increased respiration and generation of reactive oxygen species, resulting in ATP depletion, opening of mintochondrial permeability trensition, and programmed cell death. Plant Physiology, 128, $1271-1281$.

Xu ZB (徐质斌) (2000). Development prospect of seawater irrigating agriculture in Shandong Province. Development Forum (发 展论坛), 8(1), 24-25. (in Chinese)

Yan XX, Zhao TE, Hu YJ (1994). Effect of moderate salt stress on cells in root tips of barley. Acta Agriculture Boreali-Sinica, 9 (Suppl. ), $61-64$.

Yu BJ (於丙军), Luo QY (罗庆云), Liu YL (刘友良) (2001). Effects of salt stress on growth and ionic distribution of salt-born Glycinesoja. Acta Agronomica Sinica (作物学报), 27, 776 - 780. (in Chinese with English abstract)

Zhang BD (张邦定) (1997). The exploitation and planting of $\mathrm{He}$ lianthus tuberosus. Science and Technology of Agriculture in Sichuan（四川农业科技），(5)，35-37。(in Chinese)

Zhang HY (张海燕), Zhao KF (赵可夫) (1998). Effects of salt and water stresss on osmotic adjustment of Suaeda salsa seedings. Acta Botanic Sinica (植物学报), 40,56-61. (in Chinese with English abstract) 